\title{
PROCESSO DE ENFERMAGEM: ANÁLISE DE DISSERTAÇÕES E TESES DE ENFERMAGEM NO BRASIL DE 1979-2007
}

Denise Tomaz Aguiar ${ }^{1}$, Gemma Galgani Martins de Meneses ${ }^{1}$, Francisco José Maia Pinto ${ }^{2}$, Thereza Maria Moreira Magalhães², Ana Virginia de Melo Fialho²

RESUMO: O Processo de Enfermagem direciona as atividades do Enfermeiro para um cuidado sistemático. Este estudo bibliográfico e quantitativo investigou o conhecimento produzido pelos programas de pós-graduação brasileiros em Enfermagem sobre Processo de Enfermagem, de 1979 a 2007. Os dados foram coletados do CD-ROM e da página da Associação Brasileira de Enfermagem que dispõe dos catálogos do Centro de Estudos e Pesquisas em Enfermagem. Um total de 112 estudos foram submetidos a uma análise descritivo-frequencial. Percebe-se crescente número de estudos sobre a temática nos últimos anos 57 (50,9\%), com foco nos sujeitos assistidos 38(33,9\%); destaca-se que 48 (42,8\%) dos estudos utilizaram as teorias de enfermagem como referencial, e constatou-se um predomínio na etapa do diagnóstico de enfermagem. A temática apresenta crescimento na produção científica, embora em sua maioria esta fragmente o processo de enfermagem com abordagem somente de uma ou duas de suas etapas.

PALAVRAS-CHAVE: Enfermagem; Processos de enfermagem; Assistência ao usuário.

\section{NURSING PROCESS: ANALYSIS OF NURSING THESIS AND DISSERTATIONS IN BRAZIL FROM 1979 TO 2007}

\begin{abstract}
The Nursing Process directs the activities of a nurse to care routine. This quantitative study investigated the literature and knowledge produced by the nursing graduate programs about nursing process, from 1979 to 2007. Data were collected from CD-ROM and website of the Brazilian Association of Nursing who has the catalogs of the Center for Studies and Research in Nursing. A total of 112 studies were submitted to descriptive analysis-frequency. A growing number of studies on this subject over the past 57 years (50.9\%), with a focus on the subjects attended 38 $(33.9 \%)$ is stated. It stands out that $48(42.8 \%)$ studies used nursing theories as a referential, with a predominance in the stage of nursing diagnosis. The theme presents growth in scientific production but most of it shred the nursing process approaching only one or two of its stages.
\end{abstract}

KEYWORDS: Nursing; Nursing process; User assistance.

\section{PROCESO DE ENFERMERÍA: ANÁLISIS DE DISERTACIONES Y TESIS DE ENFERMERÍA EN BRASIL DE 1979-2007}

RESUMEN: El proceso de enfermería direcciona las actividades del enfermero para un cuidado sistemático. Este estudio bibliográfico y cuantitativo investigó el conocimiento producido por los programas de posgrado brasileños en enfermería acerca de proceso de enfermería, de 1979 a 2007. Los datos fueron recogidos del CD-ROM y del sitio de la Asociación Brasileña de Enfermería que dispone de los catálogos del Centro de Estudios e Investigaciones en Enfermería. Un total de 112 estudios fueron sometidos a un análisis descriptivo-frecuencial. Se percibió crecente número de estudios acerca de la temática en los últimos años - 57 (50,9\%), con foco en los sujetos asistidos - 38(33,9\%); se destaca que 48 $(42,8 \%)$ de los estudios utilizaron las teorías de enfermería como referencial; se constató un predominio en la etapa del diagnóstico de enfermería. La temática presenta crecimiento en las produccion científica, sin embargo en su mayoria fragmente el proceso de enfermería con abordaje solamente de una o dos de sus etapas.

PALABRAS CLAVE: Enfermería; Procesos de enfermería; Asistencia al usuario.

${ }^{1}$ Enfermeira. Mestranda em Cuidados Clínicos em Saúde na Universidade Estadual do Ceará-UECE.

${ }^{2}$ Enfermeiro. Doutor em Enfermagem. Docente da UECE.

Autor correspondente:

Denise Tomaz Aguiar

Universidade Estadual do Ceará

R. Recanto tranquilo, 102 - 60742-370 - Fortaleza-CE-Brasil

Recebido: 17/03/10

Email: denisetmz@yahoo.com.br Aprovado: 01/09/10 


\section{INTRODUÇÃO}

O Processo de Enfermagem (PE) surgiu a partir da preocupação de direcionar as atividades de Enfermagem baseadas em um método científico. No contexto da Sistematização da Assistência de Enfermagem (SAE). O PE se mostra como um caminho utilizado para instrumentalizar a resolução de problemas, fundamentando as ações do enfermeiro.

O PE foi, inicialmente, citado na literatura norteamericana, nas décadas de 1950 e 1960. A partir desse período, novas experiências foram adquiridas, mais conhecimentos foram desenvolvidos e os programas de ensino focalizaram o cuidado de enfermagem na identificação e solução de problemas de saúde ${ }^{(1)}$. No Brasil, o PE ganhou destaque na década de 70, a partir do modelo conceitual de Wanda de Aguiar Horta $^{(2)}$.

Para a operacionalização do PE, são sugeridas seis etapas: histórico, diagnóstico, planejamento, prescrição, intervenção e avaliação. Estas devem ser interdependentes, interligadas, complementares e dinâmicas. Além disso, para uma assistência sistematizada é imprescindível a fundamentação em uma das teorias de enfermagem, escolhidas conforme a realidade e o cenário de cada cuidado e de cada sujeito ${ }^{(3)}$

O PE constitui uma forma sistemática e dinâmica de prestar cuidados, promover a humanização e direcionar resultados, facilitando a mensuração dos custos. É essencial a todas as abordagens de Enfermagem e impulsiona os enfermeiros a estar continuamente examinado suas ações ${ }^{(4)}$.

Para classificar e nomear os fenômenos, intervenções e resultados envoltos no desenvolvimento do PE, há várias abordagens, sendo a taxonomia da NANDA (North American Nursing Diagnosis Association), a classificação das intervenções da NIC (Nursing Interventions Classification) e os resultados propostos pela NOC (Nursing Outcomes Classification) dotados de ampla aplicação mundial na área de Enfermagem. Porém, existem outras classificações, como a Classificação Internacional para a Prática de Enfermagem (CIPE), com o intuito de uniformizar e estabelecer uma linguagem comum que represente a prática de enfermagem no mundo ${ }^{(5)}$.

Para desenvolver o PE é necessário que o enfermeiro possua habilidade e competência para que, na tomada de decisão, sejam identificadas corretamente as necessidades dos usuários.

Acredita-se que o conhecimento da produção acadêmica sobre a temática auxiliará a Enfermagem a prover o cuidado de forma mais efetiva e eficaz, pois o cuidado sistematizado reflete uma preocupação atual na realização de pesquisas científicas, como também uma necessidade para enfermeiros que atuam diretamente na assistência. Dessa forma, analisar os temas pesquisados sobre o PE pelos enfermeiros do país, pode contribuir para (re)dimensionar a prática da enfermagem.

Pouco útil seria pesquisar soluções para os problemas e inquietações da prática profissional em relação ao PE se estas pesquisas não fossem compartilhadas no meio científico. A produção do conhecimento é um processo refinado e dinâmico que, após sua construção, deve ser socializado por meio de publicações em periódicos, catálogos de produção, congressos, seminários e outros meios de comunicação ${ }^{(6)}$.

Assim, este estudo teve como objetivo descrever o conhecimento produzido pelos programas de pós-graduação brasileiros em Enfermagem sobre a temática "Processo de Enfermagem", no período de 1979 a 2007.

\section{METODOLOGIA}

Trata-se de um estudo bibliográfico, quantitativo. Esse tipo de estudo abrange a bibliografia já tornada pública, em relação ao tema do estudo: publicações avulsas, boletins, jornais, revistas, livros, pesquisas, monografias, teses, material cartográfico e outros. A pesquisa bibliográfica propicia o exame de um tema sob novo enfoque ou abordagem, inovando com conclusões críticas ${ }^{(7)}$.

Os dados foram coletados do CD-ROM do Centro de Estudos e Pesquisas em Enfermagem (CEPEn) e por meio do "Catálogo de Informações sobre Pesquisas e Pesquisadores em Enfermagem", disponível para consulta e reprodução diretamente no endereço eletrônico da Associação Brasileira de Enfermagem (ABEn).

O CD-ROM do CEPEn comporta os resumos das dissertações e teses produzidas nos programas de pós-graduação stricto sensu das Escolas de Enfermagem de todo o país, compreendendo os anos de 1979 a 2000, além de um resumo do ano de 1975 que constava no banco de dados ${ }^{(8)}$. Os dados coletados no endereço eletrônico da ABEn, no "Catálogo de Informações sobre Pesquisas e Pesquisadores em Enfermagem", referem-se ao período de 2001 a $2007^{(9)}$.

O corpus do estudo foi constituído pelas 
publicações indexadas (149) disponíveis nos meios pesquisados. Foram excluídos 37 resumos que, mesmo selecionados com a utilização do descritor "processo de enfermagem", após leitura, identificou-se que não estavam relacionados à temática. Desde modo, a amostra constou de 112 estudos.

$\mathrm{O}$ instrumento de coleta de dados foi um formulário contendo: nome dos autores; título; local (instituição de ensino superior-IES); número de classificação no registro no CEPEn; ano; tipo de pósgraduação stricto sensu; tipo e/ou natureza; objetivos; fonte dos dados; e eixo teórico. Alguns desde dados nos auxiliaram na identificação e compreensão de como foi realizado o estudo. As variáveis de análise foram: ano; pós-graduação stricto sensu; local (instituição de ensino superior-IES); sexo dos autores; objetivos; fonte dos dados; e eixo teórico.

Inicialmente, realizou-se a digitação, tabulação e análise no programa estatístico Statistical Package for the Social Sciences-SPSS for Windows, versão 15.0. A análise foi realizada a partir da estatística descritiva.

Quanto aos aspectos éticos, por se tratar de uma pesquisa bibliográfica, o estudo não necessitou da avaliação por um comitê de ética em pesquisa. Também não houve necessidade de solicitar permissão do estudo à ABEn ou ao CEPEn, pois o material é uma publicação eletrônica disponível na internet, de livre acesso, facilitando a consulta e a ampliação da difusão da produção acadêmica da Enfermagem brasileira.

\section{RESULTADOS E DISCUSSÃO}

Após a análise dos resumos, foi detectado que o número total de dissertações, teses ou livre docência relativas à temática era de 112 estudos.

Tabela 1 - Tipo de pós-graduação stricto sensu relacionada ao ano dos estudos. CEPEn, 1979-2007

\begin{tabular}{|c|c|c|c|c|}
\hline \multirow[b]{2}{*}{ Período } & \multicolumn{3}{|c|}{$\begin{array}{c}\text { Tipo de Pós-Graduação } \\
\text { Stricto Sensu }\end{array}$} & \multirow[b]{2}{*}{ Total } \\
\hline & Doutorado & $\begin{array}{c}\text { Livre } \\
\text { docência }\end{array}$ & Mestrado & \\
\hline $1975|--| 1979$ & 0 & 1 & 0 & 1 \\
\hline $1980|--| 1989$ & 0 & 1 & 11 & 12 \\
\hline 1999 |--| 1990 & 8 & 1 & 33 & 42 \\
\hline $2000|--| 2007$ & 12 & 0 & 45 & 57 \\
\hline Total & 20 & 3 & 89 & 112 \\
\hline
\end{tabular}

Do total de 112 trabalhos, $1(0,9 \%)$ estudo tratava-se de tese de livre-docência e feita na década 70. A década de 90 foi marcada pelo aumento na procura pelos cursos de mestrado e doutorado, bem como pela maior preocupação com o cuidado de enfermagem, com a introdução da SAE, o que pode ser observado com o total de 42 (37\%) estudos entre mestrado, livre-docência e doutorado. De 2000 a 2007, detectou-se aumento deste número para 57 (50,9\%) estudos (Tabela 1).

O período que mais se destacou foi o de 2000 a 2007, o que pode ser um reflexo do interesse maior em relação à temática pelas instituições, e do crescente envolvimento do enfermeiro e de pesquisadores com a temática, subsidiando o ensino e a produção de conhecimentos na área.

Os resultados mostram que o maior acesso dos enfermeiros foi aos cursos de mestrado, principalmente pela maior oferta deste curso no Brasil, se comparado aos doutorados. Atualmente, a Enfermagem possui 37 cursos de mestrado, sendo 34 acadêmicos e três profissionais. Em relação aos cursos de doutorado, constatou-se 18 cursos reconhecidos e recomendados pela Coordenação de Aperfeiçoamento de Pessoal de Nível Superior (CAPES). Esses dados mostram a preocupação com o avanço científico da Enfermagem, pois o crescimento da pós-graduação traz contribuições importantes à formação profissional de qualidade ${ }^{(10)}$.

Embora a literatura publicada sobre a SAE apresente crescimento gradual desde 2000, observase ainda a necessidade de maior aproximação entre a teoria e a prática da Enfermagem. Tal distanciamento gera conflitos ideológicos que prejudicam a compreensão da prática da Enfermagem e o ensino de suas teorias, processo e metodologia da assistência ${ }^{(11)}$.

A tabela 2 traz os trabalhos distribuídos segundo região do território brasileiro.

Tabela 2 - Distribuição dos estudos por região. CEPEn, 1979-2007

\begin{tabular}{lccc}
\hline Região & F & $\mathbf{\%}$ & Fa \\
\hline Centro-Oeste & $\mathbf{2}$ & $\mathbf{1 , 8}$ & $\mathbf{1 , 8}$ \\
MG & 2 & & \\
Nordeste & $\mathbf{2 9}$ & $\mathbf{2 5 , 9}$ & $\mathbf{2 7 , 7}$ \\
CE & 8 & & \\
CE/MA & 1 & & \\
PB & 16 & & \\
BA & 3 & & \\
RN & 1 & & \\
\hline
\end{tabular}

Continua 
Continuação

\begin{tabular}{lccc}
\hline Região & F & \% & Fa \\
\hline Sudeste & $\mathbf{5 6}$ & $\mathbf{5 0 , 0}$ & $\mathbf{7 7 , 7}$ \\
RJ & 13 & & \\
SP & 43 & & \\
Sul & $\mathbf{2 5}$ & $\mathbf{2 2 , 3}$ & $\mathbf{1 0 0 , 0}$ \\
SC & 20 & & \\
PR & 2 & & \\
RS & 3 & & \\
\hline Total & $\mathbf{1 1 2}$ & $\mathbf{1 0 0 , 0}$ & \\
\hline
\end{tabular}

Os resumos identificados sobre a temática estiveram distribuídos em 19 Escolas de Enfermagem, sendo que a metade, 56 (50\%), foi da região sudeste. Esta concentração de estudos se dá por existir grandes centros de pós-graduação, geradores de conhecimento; percebe-se grande produção na Paraíba.

Outro aspecto levantado foi o das fontes de dados (composta por sujeitos que participaram das pesquisas, prontuários, documentos, dentre outros) utilizadas na coleta de informações durante os estudos (Tabela 3).

Tabela 3 - Fontes de dados pesquisadas nos estudos. CEPEn, 1979-2007

\begin{tabular}{lccc}
\hline Fontes dos dados & F & $\mathbf{\%}$ & Fa \\
\hline Enfermeiro assistencial & 22 & 19,6 & 19,6 \\
Enfermeiro docente & 5 & 4,5 & 24,1 \\
Usuário & 38 & 33,9 & 80,4 \\
Prontuário & 10 & 8,9 & 89,3 \\
Outros & 25 & 22,3 & 46,4 \\
Sem referência & 12 & 10,7 & 100,0 \\
\hline Total & $\mathbf{1 1 2}$ & $\mathbf{1 0 0 , 0}$ & \\
\hline
\end{tabular}

A maioria dos estudos, 75 (78\%), envolveu: os usuários, em 38 (33,9\%), e os enfermeiros, em 27 $(24,1 \%)$ (Tabela 3). Entre os enfermeiros, 22 (19,6\%) eram assistenciais e $5(4,5 \%)$ eram docentes. Esses dados expressam o maior enfoque dado aos usuários por serem o alvo da SAE, e aos enfermeiros assistenciais por serem o foco de sua implementação.

A utilização da sistematização da assistência deve ser individualizada e centrada nas necessidades do usuário, proporcionando aos profissionais mais segurança na tomada de decisão no processo de trabalho e oferecendo benefícios aos usuários e profissionais ${ }^{(3)}$. A SAE eleva a qualidade da assistência de enfermagem $^{(5)}$.
Houve predomínio do sexo feminino 106 (95\%) entre os autores, o que está relacionado à caracterização da profissão como eminentemente feminina. Essa questão é mencionada em qualquer estudo que trate de aspectos relativos à identidade profissional, ao gênero ou à história da profissão.

Outra variável de análise foi a dos referenciais teóricos utilizados, considerados instrumentos indispensáveis à operacionalização do processo de enfermagem (Tabela 4). A escolha de um referencial teórico direciona a construção do instrumento e a coleta de dados, pois cada teoria apresenta contexto específico para determinada situação ${ }^{(12)}$.

Tabela 4 - Eixos teóricos utilizados nos estudos. CEPEn, 1979 a 2007

\begin{tabular}{lccc}
\hline Eixos teóricos estudados & F & $\mathbf{\%}$ & Fa \\
\hline Teoria de Madeleine Leninger & 3 & 2,7 & 2,7 \\
Teoria de Sister Calistta Roy & 3 & 2,7 & 5,4 \\
Teoria de Dorothea Orem & 5 & 4,5 & 9,9 \\
Teoria de Wanda Horta & 23 & 20,5 & 30,4 \\
Outras teorias de enfermagem & 14 & 12,5 & 42,9 \\
Outros eixos teóricos & 14 & 12,5 & 55,4 \\
Sem referência & 50 & 44,6 & 100,0 \\
\hline Total & $\mathbf{1 1 2}$ & $\mathbf{1 0 0 , 0}$ \\
\hline
\end{tabular}

Houve predominância da escolha e utilização de um referencial teórico da enfermagem que não se adaptava ao foco de trabalho $(57,1 \%)$ (Tabela 4). Diante dos estudos analisados, nota-se que a maior parte, $50(44,6 \%)$, não apresentava referencial e $14(12,5 \%)$ se fundamentaram em outros eixos não relativos às teorias de enfermagem, como: Martin Heidegger, Avaliação Emancipatória, Pedagogia da Problematização, Gestalt Terapia, dentre outros.

Ressalta-se que 48 (42,8\%) dos estudos utilizaram teorias de enfermagem, sendo a mais utilizada a de Wanda Horta, em 23 (20,5\%) dos estudos. Outras teoristas de enfermagem utilizadas foram Orem, Leininger, Imogene King, Travelbee, Nightingale, Paterson e Zderard, sendo também utilizado como referencial a taxonomia da NANDA.

A aplicação da sistematização da assistência com base no PE, e sustentada por uma teoria de enfermagem, melhora a qualidade dos cuidados prestados, humaniza o atendimento, define o papel do enfermeiro, dá autonomia à profissão, direciona a equipe e facilita a mensuração dos custos das instituições. Em contrapartida, aumenta a responsabilidade dos 
profissionais quanto aos cuidados prestados aos usuários e exige conhecimento científico mais profundo e específico ${ }^{(13)}$.

Quadro 1 - Aspectos do Processo de Enfermagem contidos nos resumos. CEPEn, 1979-2007
O quadro 1 aborda os aspectos presentes nos resumos relativos ao $\mathrm{PE}$.

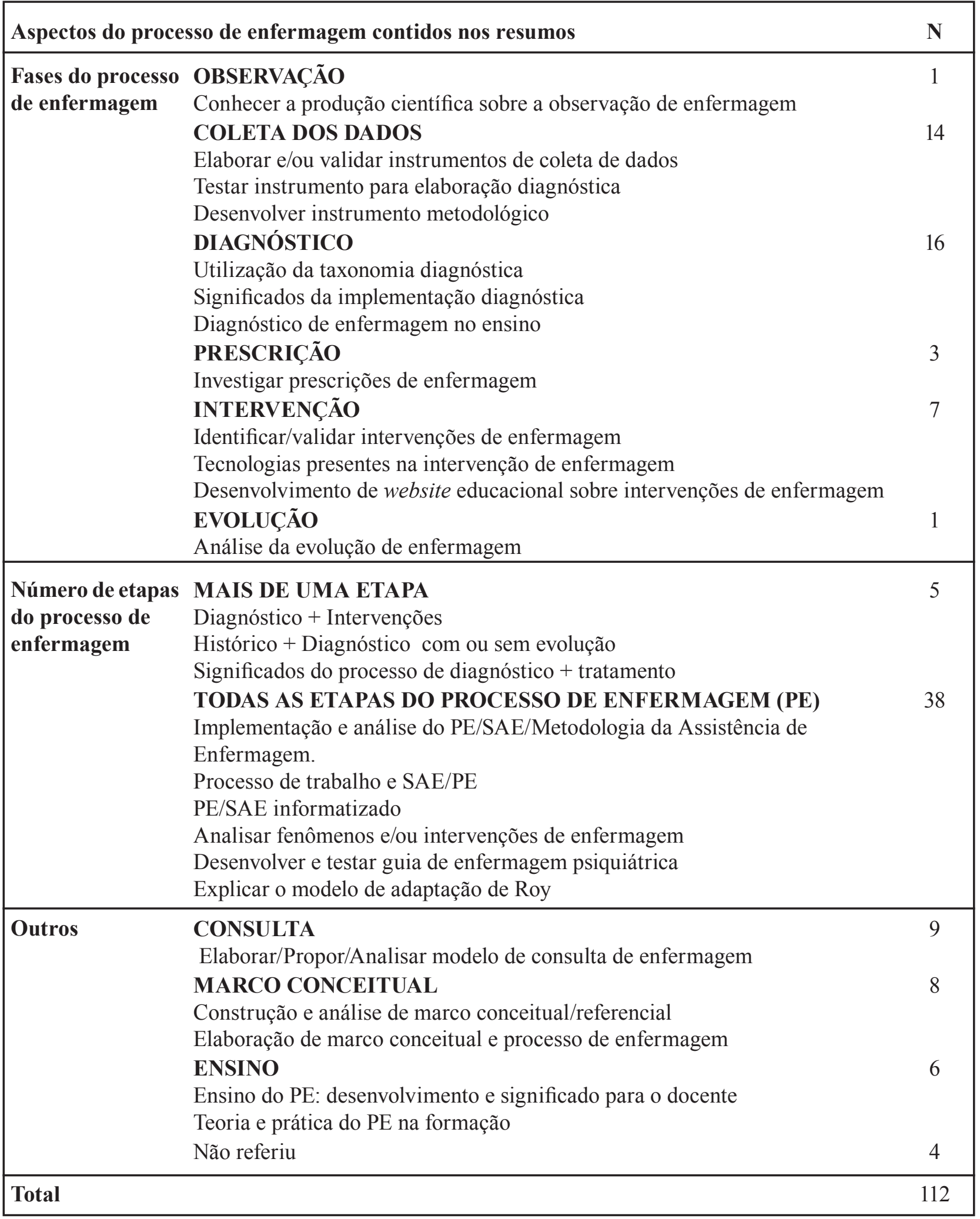


Assim, percebe-se que o PE perde sua essência ao ser abordado de forma fragmentada, o que se deu na maioria dos estudos, 74 (66 \%). Estes não abordaram as seis etapas integradas do PE, mas apenas algumas etapas separadas, independentes e fragmentadas. Dentre estes fragmentos do $\mathrm{PE}$, a maior parte dos resumos, 16 (14,3\%), se referiu ao diagnóstico de enfermagem, denotando a preocupação dos profissionais em trabalhar com respostas humanas a processos e quadros patológicos, numa visão ainda reducionista do ser humano, sobretudo no ambiente hospitalar, quando aquele se encontra isolado de sua família e contexto.

O estudo da etapa de anamnese ou coleta de dados foi abordado em 14 resumos (12,5\%). Esta etapa é uma das mais importantes, por iniciar o PE, e precisa ser adequadamente planejada com o intuito de minimizar eventuais falhas e garantir maior êxito à sistematização da assistência. O que deve ser buscada nesta etapa é a preocupação com um instrumento não mecânico, acrítico e padronizado para todos os usuários, mas com a utilização de uma proposta flexível, reflexiva e que considere o sujeito de forma singular. A complexidade humana, pela qual cada indivíduo apresenta especificidades, singularidades e multidimensionalidades durante o cuidado de enfermagem, é justificativa para tal exigência ${ }^{(14)}$.

\section{CONCLUSÃO}

Com esse estudo constata-se que, no período de 1979 a 2007, a temática "Processo de Enfermagem" apresentou crescimento evidente nas produções científicas, com diversidade de abordagens, sendo que a maioria destas se dá de forma fragmentada, não envolvendo todas as etapas integradas e dinâmicas do PE, como rege sua proposta na essência.

Os resumos analisados mostraram-se preocupados com o sujeito como foco de atenção do processo, sobretudo voltados para diagnósticos de enfermagem.

O PE é uma das formas de buscar cientificidade para a profissão. No entanto, foi evidente a necessidade de uma reflexão crítica acerca de sua operacionalização, considerando a individualidade de cada sujeito, pois o sujeito assistido na sua singularidade e no seu contexto histórico-cultural deve ser inserido como principal colaborador do processo saúde-doença-cuidado.

\section{REFERÊNCIAS}

1. Leon PAP, Freitas FFQ, Nóbrega MML. Sistematização da assistência de enfermagem em dissertações de mestrado: estudo bibliográfico. Rev Enferm UFPE. 2009;3(1):120-6

2. Domingues CO, Amestoy SC, Santos E. Sistematização da assistência de enfermagem. In: XV Congresso de Iniciação Científica: 2006. Pelotas, Brasil; 2006.

3. Horta WA. O processo de enfermagem. São Paulo: EPU/EDUSP; 1979.

4. Alvaro-Lefevre R. Aplicação do processo de enfermagem: promoção do cuidado colaborativo. $5^{\text {a }}$ ed. Porto Alegre: Artmed; 2005.

5. Nóbrega MML, Silva KL. Fundamentos do cuidar em enfermagem. $2^{\text {a }}$ ed. Belo Horizonte: ABEN, 2008/2009.

6. Costa RS, Carvalho DV. Análise da produção científica dos enfermeiros de Minas Gerais publicada em periódicos de enfermagem. Rev Latino-Am Enfermagem. 2001;9(5):19-25.

7. Lakatos EM, Marconi MA. Fundamentos de metodologia científica. $6^{\mathrm{a}}$ ed. São Paulo: Atlas; 2007.

8. Associação Brasileira de Enfermagem (ABEn). Informações sobre pesquisas e pesquisadores em enfermagem - Centro de Estudos e Pesquisa em Enfermagem [CD-ROM]. Brasília (DF): ABEn; 2001.

9. Centro de Estudos e Pesquisa em Enfermagem (CEPEn). Informações sobre pesquisas e pesquisadores em enfermagem. Associação Brasileira de Enfermagem. 2001-2004 [acesso em 06 maio 2009]. Disponível: http://www.abennacional.org.br

10. Coordenação de Aperfeiçoamento de Pessoal de Nível Superior (CAPES). [acesso em 11 jun 2009]. Disponível: www.capes.gov.br

11. Fuly PSC, Leite JL, Lima SBS. Correntes de pensamento nacionais sobre sistematização da assistência de enfermagem. Rev Bras Enferm. 2008; 61(6): 883-7.

12. Carvalho EC, Rossi LA. Modelos, estruturas e teorias de enfermagem: aplicação através do processo de enfermagem. In: Garcia TR, Pagliuca LMF, coordenadores. A construção do conhecimento em enfermagem: coletânea de trabalhos. Fortaleza (CE): 
RENE; 1998. p 65-85.

13. Tannure MC, Gonçalves AMP. SAE - sistematização da assistência de enfermagem: guia prático. Rio de Janeiro: Guanabara Koogan, 2008

14. Lopes FL, Szewczyk MSC, Lunardi VL, Santos SSC. SAE como um novo fazer na atividade cuidativa da enfermagem com base na complexidade de Edgar Morin. Cogitare Enferm. 2007;12(1):115-9. 